Part of Journal of Research of the National Bureau of Standards, Volume 23, September 1939

\title{
AN APPARATUS FOR MAGNETIC TESTING AT MAGNETIZ- ING FORCES UP TO 5,000 OERSTEDS
}

\author{
By Raymond L. Sanford and Evert G. Bennett
}

\section{ABSTRACT}

An apparatus is described for general magnetic testing in the range of values of magnetizing foree from 100 to 5,000 oersteds. Specimens of rectangular cross section up to $3.8 \mathrm{~cm}\left(1 \frac{1}{2}\right.$ in.) wide and $1.9 \mathrm{~cm} \mathrm{(3/4} \mathrm{in.)} \mathrm{thick} \mathrm{can} \mathrm{be} \mathrm{tested.} \mathrm{It} \mathrm{is}$ estimated that under favorable conditions values of induction or magnetizing force accurate within 0.5 percent can be obtained and that under ordinary conditions of routine testing the accuracy is within 1 percent. The apparatus is convenient and simple to operate and does not heat the specimen.

CONTENTS

I. Introduction............ 415

II. Description of apparatus

III. Experimental investigation _.

IV. Accuracy of test data

V. Summary _.

\section{INTRODUCTION}

Previous to 1920, when Honda and Saito announced their KS magnet steel, ${ }^{1}$ a maximum magnetizing force of 300 oersteds was adequate for the testing of permanent-magnet steels. However, cobalt magnet steels of the KS type, and others which were developed soon after, required a maximum magnetizing force of 1,000 oersteds, and consequently new permeameters were needed. The need was fairly well met by the Babbitt permeameter, ${ }^{2}$ the Full-Range permeameter, ${ }^{3}$ and an apparatus developed by the present authors. ${ }^{4}$

In 1932 Mishima ${ }^{5}$ announced an entirely new type of permanentmagnet alloy containing aluminum and nickel as the essential alloying elements. Following this, several alloys of similar characteristics, including the alloy known as Alnico, ${ }^{6}$ have been produced, which are magnetically harder than the earlier permanent magnet materials and consequently require higher magnetizing forces for testing. The authors were able to extend the range of their apparatus to 2,000 oersteds by the addition of auxiliary magnetizing coils, but a further

1 Honda and Saito, On KS magnet steel, Phys. Rev. 16, pt. 2, 495 (1920).

2 B. J. Babbitt, An improved permeameter for testing magnet steel, J. Opt. Soc. Rev. Sei. Instr. 1\%, 47 (1928)

3 B. M. Smith, A new full-range permeameter, Gen. Elec. Rev. 38, 520 (1935).

1 Sanford and Bennett, An apparatus for magnetic testing at high magnetizing forces, BS J. Research 10 , 567 (1933) RP548.

\$ T. Mishima, Magnetic properties of iron-nickel-aluminum alloys, Ohm (July 1932); Iron Age (abstract) 130, 346 (1932).

6J. Q. Adams, Alnico-its properties and possibilities, Gen. Elec. Rev. 41, 518 (1938). 
increase was impracticable because the magnetic circuit had too small a cross section.

In view of the increasing use of the new materials and the possibility that magnetizing forces higher than 2,000 oersteds might be needed before long, the development of a new permeameter was undertaken. It was hoped that an instrument could be produced which would be capable of applying magnetizing forces up to 5,000 oersteds and of giving a better accuracy than the previous instrument.

The final result was the "High- $H$ permeameter" shown in figure 2 . This apparatus is capable of testing specimens up to $3.8 \mathrm{~cm}\left(1 \frac{1}{2} \mathrm{in}\right.$.) wide and $1.9 \mathrm{~cm}(3 / 4$ in.) thick. At magnetizing forces from 100 to 5,000 oersteds, values of either magnetizing force or of induction can be measured with an error not greater than 1 percent. Measurements have been made at magnetizing forces as high as 9,000 oersteds without appreciable heating, but the accuracy at these higher forces has not been determined.

\section{DESCRIPTION OF APPARATUS}

In designing the present apparatus, essentially the same principles were followed as in the case of the earlier one. The principal modifications were made for the purpose of increasing the range and improving the accuracy of the measurements. Certain features were also added in order to provide flexibility and convenience in operation.

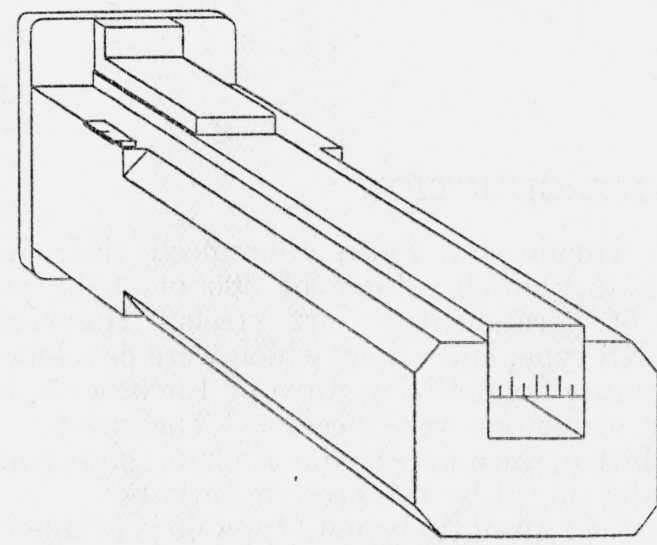

Figure 1.-Pole-piece

To prevent undue heating of the specimen, a magnetic circuit of the isthmus type was adopted, as the necessary high magnetizing forces can thus be applied using only a moderate amount of power. This type of circuit, involving only a relatively short length of specimen, has the added advantage that specimens of ordinary length can easily be examined for uniformity-an important point if specimens are to be used for the intercomparison of permeameters. Furthermore, it has been found possible to obtain satisfactory results on a specimen only $2.5 \mathrm{~cm}$ ( 1 in.) long, which is too short to be tested in other existing permeameters.

Saturation effects have been reduced by substantially increasing the cross sections of the yokes and pole pieces, which form a symmetrical magnetic circuit.

If values of magnetizing force are obtained by means of a stationary $H$-coil, the errors in the determination of points on a hysteresis loop may be large, because it is necessary to calculate the values as the difference between two relatively large quantities. This difficulty has been overcome by the use of a rotating $H$-coil (flip-coil), by means of which the value of magnetizing force is obtained directly. 


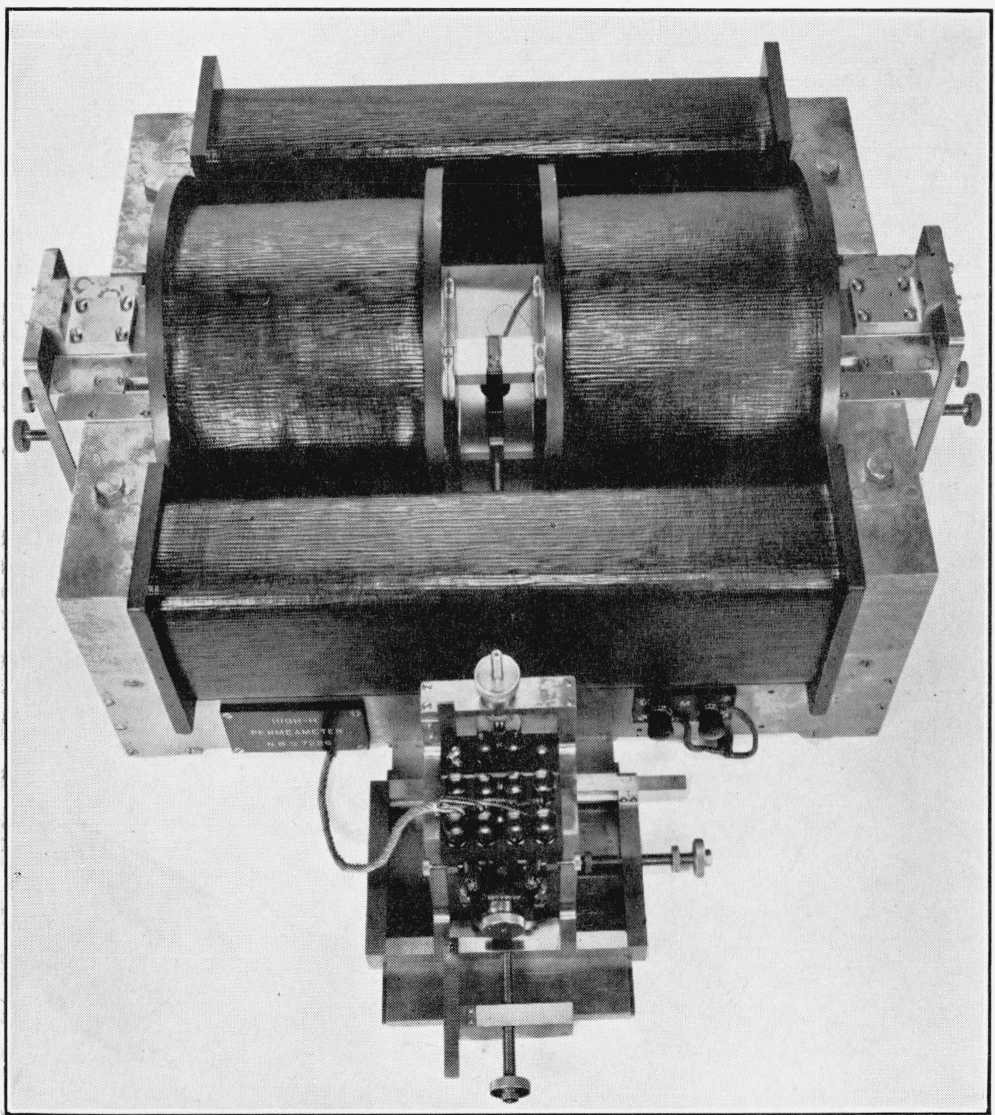

FiguRe 2.-The High-H permeameter. 


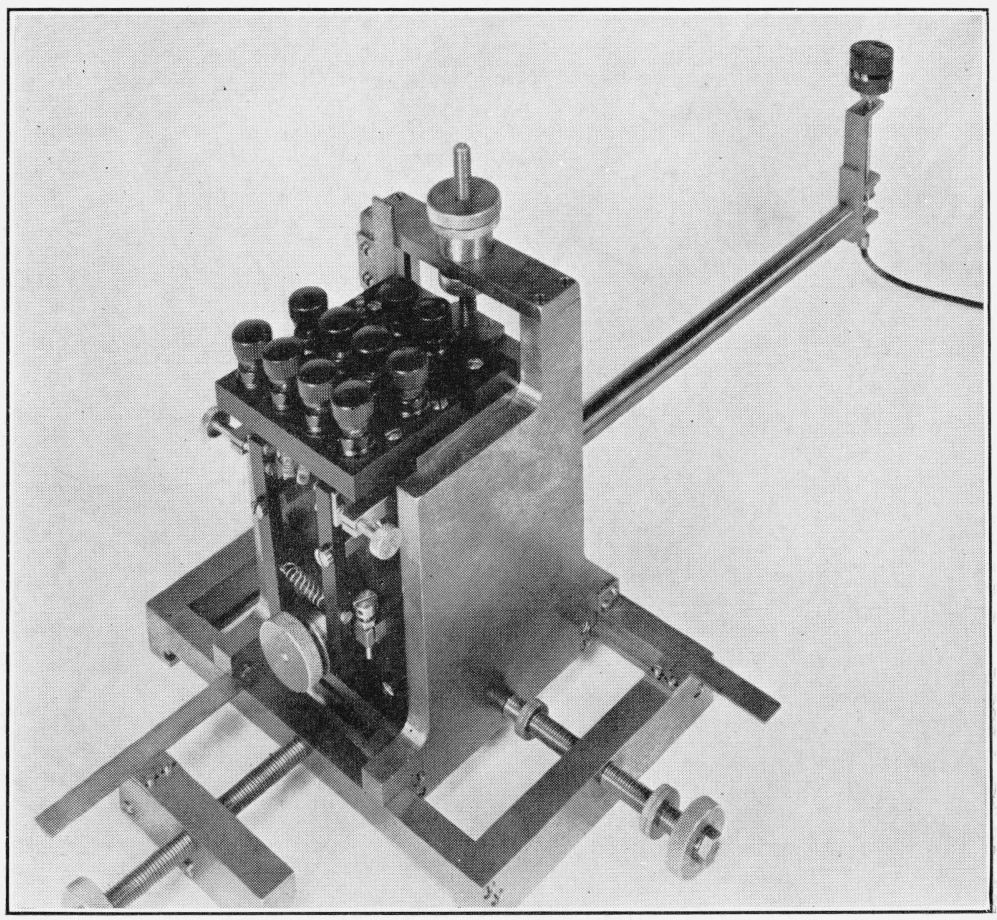

Figure 3.-Rotating H-coil and accessory apparatus. 
The magnetic circuit is shown in figure 2. The specimen is held in pole pieces extending between two $U$-shaped yokes. The construction of the pole pieces is indicated in the sketch of figure 1. They have longitudinal channels into which are fitted filler pieces as shown. The specimen is located in the space between the bottom of the channel and the filler. The magnetic circuit is constructed of laminations of No. 16 gage armature-grade silicon steel. The distance between the pole pieces, or gap length, is adjustable; and scales are provided to indicate the gap length up to $10 \mathrm{~cm}$, which is the maximum generally used. Scales are also located on the inner ends of the filler pieces to aid in centering the specimen. The filler pieces are fastened at their outer ends to heavy brass plates which are clamped to the ends of the pole pieces so as to keep the fillers parallel with the bottom of the channels.

The main magnetizing coils are on the pole pieces, and auxiliary windings are located on the yokes. The design of the magnetic circuit and magnetizing windings was greatly facilitated by preliminary experiments with a small model, by means of which the probable distribution of flux in the magnetic circuit was approximately determined. The principal dimensions and constants of the apparatus are given in table 1.

TABLE 1.-Constants of the apparatus

\begin{tabular}{|c|c|}
\hline \multicolumn{2}{|l|}{ Magnetic circuit } \\
\hline $\begin{array}{l}\text { Over-all length } \\
\text { Over-all width } \\
\text { Cross section of yokes } \\
\text { Length of pole pieces. } \\
\text { Cross section of pole pieces. } \\
\text { Width of channels. } \\
\text { Depth of channels } \\
\text { Thickness of filler pieces }\end{array}$ & $\begin{array}{l}50 \mathrm{~cm} . \\
40 \mathrm{~cm} \\
5 \times 8 \mathrm{~cm} \\
25 \mathrm{~cm} \\
8 \times 10 \mathrm{~cm} \\
4 \mathrm{~cm} . \\
4 \mathrm{~cm} \\
3 \mathrm{~cm}\end{array}$ \\
\hline \multicolumn{2}{|l|}{ Magnetizing coils } \\
\hline $\begin{array}{l}\text { No. } 14 \text { A W G silk-enamel throughout } \\
\text { Length, main coils } \\
\text { Inside diameter, main coils } \\
\text { Number of layers, main coils. } \\
\text { Number of turns, main coils } \\
\text { Length, yoke coils } \\
\text { Number of layers, yoke coils. } \\
\text { Number of turns, yoke coils } \\
\text { Total number of turns }\end{array}$ & $\begin{array}{l}15 \mathrm{~cm} . \\
11.4 \mathrm{~cm} . \\
30 . \\
2,690 \text { each. } \\
38 \mathrm{~cm} . \\
7 . \\
1,600 \text { each. } \\
8,580 .\end{array}$ \\
\hline \multicolumn{2}{|l|}{$H$-coils } \\
\hline $\begin{array}{l}\text { Length } \\
\text { Over-all width } \\
\text { Over-all thickness } \\
\text { Cross section, coil forms... } \\
\text { Turns, No. } 44 \text { AW G, enamel } \\
\text { Approximate area-turns. }\end{array}$ & $\begin{array}{l}7 \mathrm{~mm} \text {. } \\
11 \mathrm{~mm} \text {. } \\
2.5 \mathrm{~mm} \text {. } \\
0.5 \times 9 \mathrm{~mm} \text {. } \\
1,200 . \\
140 \mathrm{~cm} .^{2}\end{array}$ \\
\hline
\end{tabular}

In view of the high induced voltage resulting from the reversal of the magnetizing current, care was taken to provide ample insulation and spacing of the conductors to avoid breakdown of the insulation. The coil forms and flanges are of bakelite. As a precaution against undue arcing at the switch contacts, the apparatus is shunted with a 500 -ohm resistor. 
The general arrangement of the $H$-coil system is shown in figure 3 . There are two similar coils mounted one above the other at the top of a hollow vertical shaft so that their axes are parallel to that of the specimen. This vertical shaft can be rotated by a horizontal shaft through small beveled gears. The horizontal shaft is $32 \mathrm{~cm}$ long and is driven through spur gears by a small series-wound motor. The motor is enclosed in a soft-iron box. Adjustable stops limit the rotation of the coils to $180^{\circ}$. By means of a cam-operated switch, resistance is inserted in the motor circuit at the proper time to prevent excessive jar. The resistance is adjusted so that when it is in the circuit the motor still exerts enough torque to bring the coils back to their proper position if rebound from the stops occurs. The operating switch opens automatically so as to prevent overheating of the motor.

The carriage which supports the flip-coil assembly is mounted on ways so that its position can be adjusted in three directions by means of screws. The position of the test coils in the gap is indicated by scales graduated in millimeters. This arrangement has been found very convenient for determining field distribution in the gap. The constants of the test coils are given in table 1 under the heading $H$-coils. The coils are connected to the leads through small brass blocks held in place by screws and so located as to avoid extra loops in the test-coil circuit. This facilitates removal of the coils from the mounting for replacement or calibration.

In order to keep the air correction small, individual $B$-coils are wound on thin brass forms to fit specimens of various sizes. The coils are $5 \mathrm{~mm}$ long and have 25 turns.

The ballistic galvanometer has a period on open circuit of 45 seconds and an external resistance for critical damping of about 40,000 ohms. As the external resistance rarely exceeds 200 ohms, the behavior of the galvanometer approximates that of a fluxmeter. The galvanometer is connected by means of a selector switch to the desired test coil through any one of a number of resistors. The sensitivity is adjusted in the usual way, using a standard mutual inductor, the calibrating current being measured by a Brooks deflection potentiometer and standard shunts.

\section{EXPERIMENTAL INVESTIGATION}

In order to obtain accurate results, it is important that the induction in the specimen shall be uniform throughout the length covered by the $B$-coil and that it be possible to determine accurately the axial component of the field adjacent to the surface of the specimen over the same length. It is also desirable to know how the distribution of induction and field is affected when the gap length is varied. Accordingly, a large number of measurements were made to determine these distributions.

Most of the measurements of distribution were made using a bar of Alnico prepared by the General Electric Co. A hole approximately 8 $\mathrm{mm}$ in diameter extended longitudinally along its axis. Thus it was possible to determine the value of the field within the specimen at any point along its length by means of a test coil inserted in the hole.

The principal data obtained were longitudinal distribution of induction in the specimen, longitudinal distribution of the axial com- 
ponent of the field within the specimen, longitudinal distribution of the axial component of the field outside the specimen, and radial distribution of the axial component of the field. By radial distribution is meant the variation of the axial component of the field with the vertical distance from the bottom surface of the specimen midway between the faces of the pole pieces. The test coil used for measurements of the field outside the specimen was $5 \mathrm{~mm}$ long, $5 \mathrm{~mm}$ wide,

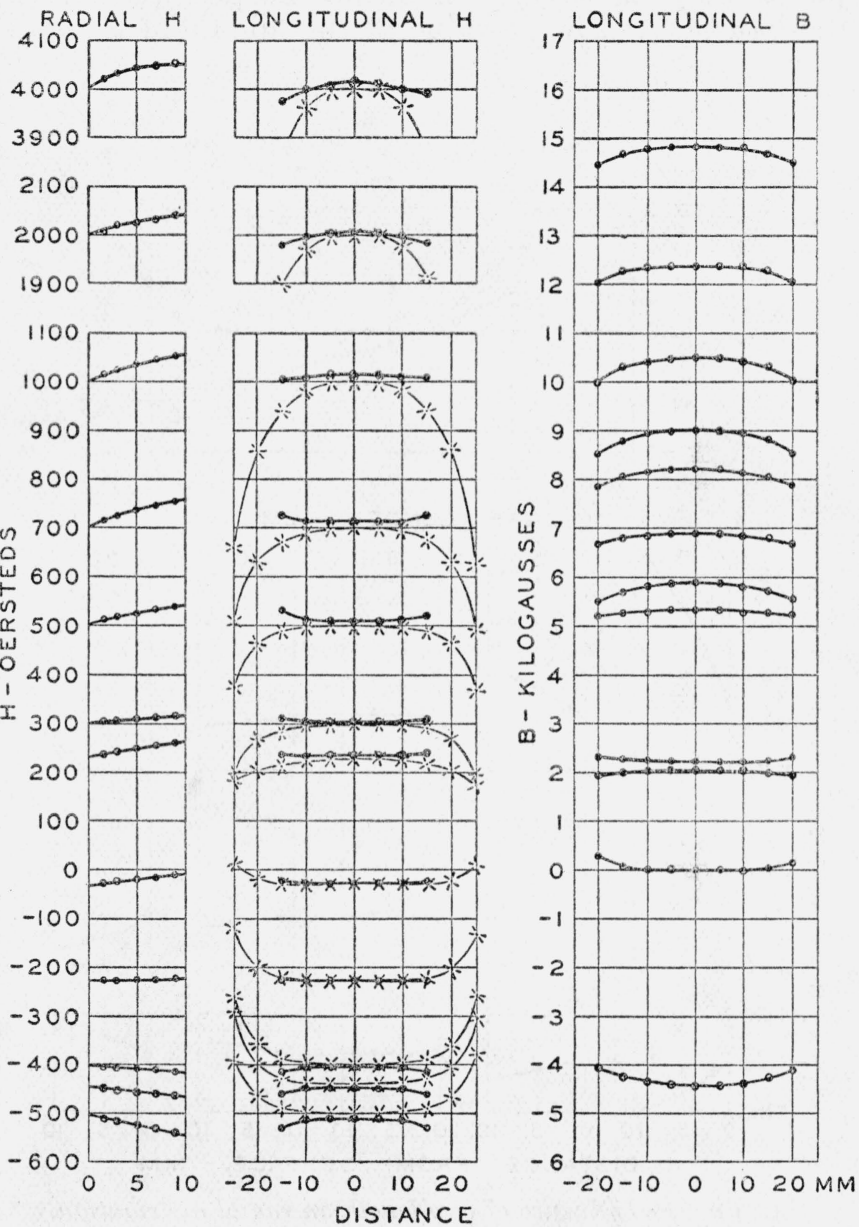

Figure 4.-Typical distribution data taken with a 5-cm gap.

and $3.2 \mathrm{~mm}$ thick. Longitudinal distribution of the field outside the specimen was obtained with the axis of the coil $1.6 \mathrm{~mm}$ below the surface. In addition to the distribution data, the influence of variations in experimental conditions, such as length of the specimen, contact reluctance at the pole pieces, straightness of the specimen, etc., was determined.

The curves shown in figure 4 are typical of the distribution data obtained and represent the results for a $5-\mathrm{cm}$ gap. The curves of 
radial $H$ show that for this length of gap the field increases with distance from the surface of the specimen. Under these conditions, a test coil of any practical dimensions calibrated in a uniform field would indicate too high values of magnetizing force. The curves of longitudinal $H$, indicated by crosses, are for the field inside the bar. This field has its highest value at the middle of the gap and decreases toward the pole pieces. Observations showed that the field is very nearly zero at points only about $2.5 \mathrm{~cm}$ inside the pole pieces. The field outside the bar has its lowest value at the middle of the gap and in-
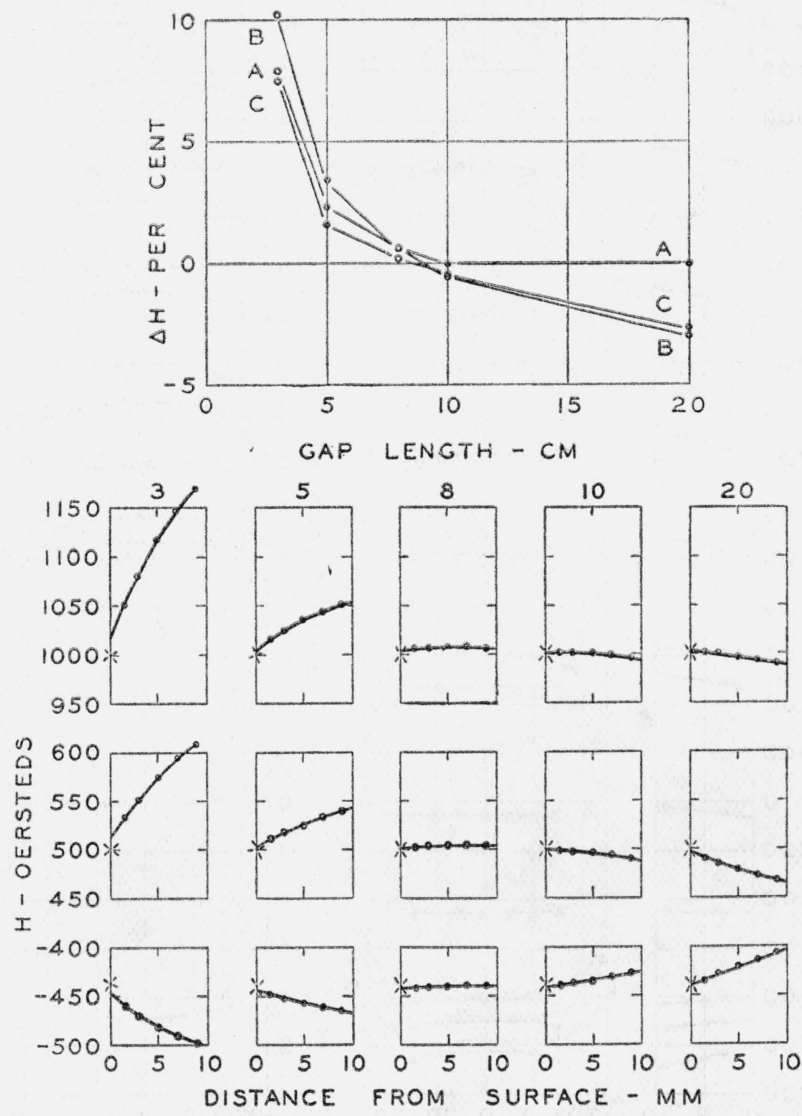

FIGURE 5.-Influence of gap length on radial distribution.

$\Delta H$ shown in upper part is the difference between the true value of $H$ and the value at a distance of $3 \mathrm{~mm}$ from the surface of the specimen. Crosses in lower part indicate the values inside the specimen.

creases toward the pole pieces. The curves of longitudinal $B$ have their highest value at the middle of the gap. The induction is very nearly constant over the middle centimeter.

Figure 5 shows how the radial distribution varies with the gap length. The lower part of the figure shows the radial distributions corresponding to fields of $1,000,500$, and -450 oersteds, respectively, measured inside the bar at the middle. The value -450 corresponds to a point on the hysteresis loop determined from a maximum magnetizing force of 1,000 oersteds. The crosses indicate the values 
obtained inside the specimen. It will be noted that the extrapolated values for the 3-cm gap are higher than the values obtained inside the specimen. This indicates that the distribution of induction is not uniform across the section for this short gap. The variation of $H$ with distance from the surface becomes less as the gap length is increased. For a gap of $8 \mathrm{~cm}$ the field is practically uniform over a distance of $1 \mathrm{~cm}$. For longer gaps the field decreases with distance from the surface.

In the upper part of figure 5 are plotted the percentage differences between the value of $H$ at a distance of $3 \mathrm{~mm}$ from the surface and
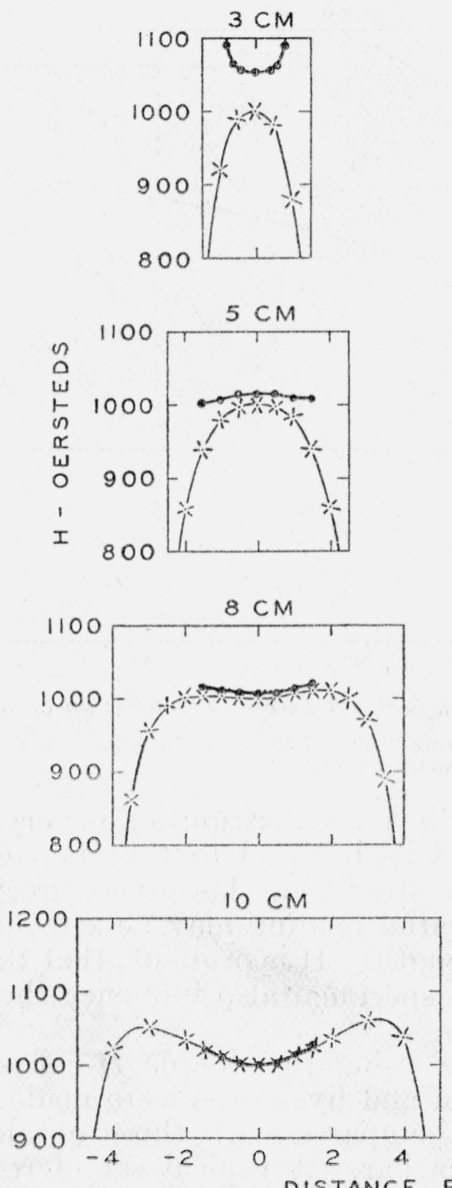
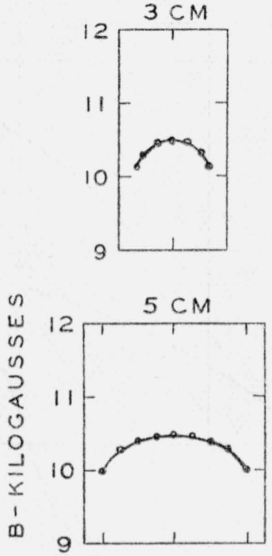

$8 \mathrm{CM}$
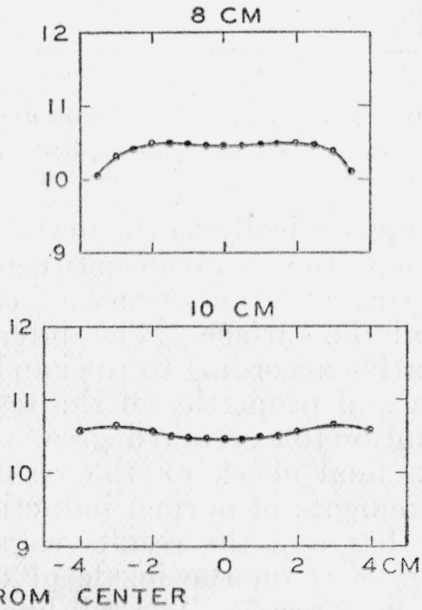

FIGURE 6.-Influence of gap length on longitudinal distribution of $H$ and $B$.

Crosses indicate values of $H$ inside the specimen. The gap lengths are 3, 5, 8, and $10 \mathrm{~cm}$, respectively.

the value inside the specimen as a function of the length of gap. Curves $A, B$, and $C$ represent the values for $1,000,500$ and -450 oersteds, respectively. The minimum difference for all values of $H$ appears to come at a gap length of about $8 \mathrm{~cm}$. Figure 6 shows the variations in the longitudinal distributions of $H$ and $B$ with the length of gap. 
The principal thing to be observed is that for a distance of about $1 \mathrm{~cm}$ at the middle of the specimen the distribution is satisfactory except for the 3-cm gap.

The distribution data were used as a guide for the design of the final $H$-coil system. The two $H$-coils are mounted with their axes parallel and $2.5 \mathrm{~mm}$ apart. The height of the system is set so that the axis of the upper coil is $2.5 \mathrm{~mm}$ away from the lower surface of the specimen. In order to determine the value of $H$ at the surface, readings are taken first with the upper coil and then with the two coils connected in series-opposition. The second reading is then

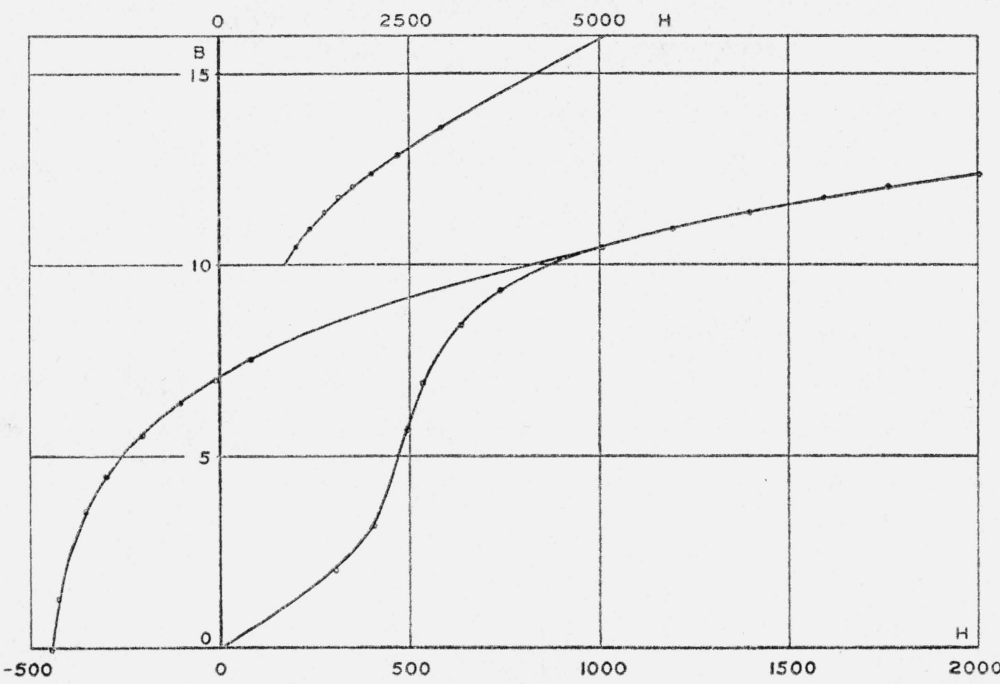

FIGURE 7.-Normal induction and hysieresis curves for hollow specimen.

Values of $H$ for the curves were obtained inside the specimen. Points refer to values taken outside the specimen. Gap length, $8 \mathrm{~cm}$.

added algebraically to the first. Since the distribution is very nearly linear and the distance between coils is equal to that between the upper coil and the surface of the specimen, this gives directly the value at the surface. The differential reading may be zero, positive or negative according to the gap length. It is probable that the cross section and properties of the test specimen also infuence the radial distribution to a certain degree.

As a final check on the results using the double $H$-coil system, measurements of normal induction and hysteresis were made on the hollow bar and the results were compared with those obtained by measuring $H$ on the inside of the bar. A typical set of results is shown in figure 7 . The full lines represent the values obtained with the internal $H$-coil, and the points were obtained with the external coils. The data obtained from measurements of the field outside the bar agree with those obtained from measurements inside the bar within the experimental error for either a 5-cm or an 8-cm gap. Results obtained with a $3-\mathrm{cm}$ gap did not agree so well, but the discrepancies did not exceed about 2 percent. 
Measurements on several solid bars having different dimensions showed good agreement between data obtained with a 5-cm gap and those for an 8-cm gap.

Experiments to determine the influence of variations in experimental conditions led to the following conclusions:

1. Ordinary variations in contact reluctance at the pole pieces do not appreciably affect the accuracy of the results.

2. The accuracy of the results does not depend upon the thickness of the specimen. The same values were obtained on a specimen having a cross section approximately 0.6 by $1.3 \mathrm{~cm}$ tested in the ordinary position and with its narrow side toward the $H$-coil.

3. Satisfactory results can be obtained with relatively short specimens. For specimens $5 \mathrm{~cm}$ or less in length, it is better to butt the ends against the pole pieces rather than to attempt to insert them in the regular way. Results agreeing within the observational error were obtained on the same section of a specimen originally $25 \mathrm{~cm}$ long after it had been cut to lengths of 10,5 , and $2.5 \mathrm{~cm}$, respectively.

4. Moderate deviations from straightness do not affect the accuracy of the results. If a bar is not straight, it is somewhat better to insert it in the apparatus with the concave side down.

5 . Unless the radial gradient is practically zero, an error in the observed value of induction may result from the use of a $B$-coil of too great area. It is best to use a $B$-coil having an area not more than 50 percent greater than that of the specimen.

The value of area-turns of the $H$-coil is too small to give satisfactory sensitivity at magnetizing forces below about 100 oersteds. For this reason no attempt has been made to determine the accuracy for lower magnetizing forces. If at some future time it should appear desirable to use the apparatus for measurements with magnetizing forces below 100 oersteds, the feasibility of using larger $H$-coils would have to be investigated.

\section{ACCURACY OF TEST DATA}

In order to estimate the accuracy of test data obtained with the apparatus, it is necessary to consider not only sources of error peculiar to the individual apparatus but also those inherent in ballistic methods in general. The principal points common to all ballistic methods include: value of the mutual inductor; area-turns of the test coils; adjustment of galvanometer sensitivity; characteristics of the galvanometer; temperature effects; uniformity of the specimen; magnetic viscosity; and observational errors. Points peculiar to the individual apparatus include: dimensions of the test coils; and method of measuring $H$ for points on the hysteresis loop.

The values of mutual inductors such as are used in connection with permeameters are generally determined to an accuracy of about \pm 0.1 percent. However, we have compared ${ }^{7}$ the inductors used with the present apparatus with a mutual inductor constructed for use in the determination of electrical resistance in absolute units under the direction of $\mathrm{F}$. Wenner with an estimated accuracy of \pm 0.05 percent.

The area-turns of the test coils were determined by measuring the mutual inductance between the coils and a standard solenoid within

7 The method used is described in Cir. NBS C415, p. 20 (1937). $168819-39-7$ 
which the coils were placed. This solenoid is wound on a threaded form and its constant, $H / I$, is known to about 0.05 percent. The distribution of field along the axis of this coil has been explored experimentally and varies by less than 0.05 percent from the calculated values. It is important in determining area-turns that the axes of the test coil and solenoid be in alinement. This was checked experimentally. It is estimated that the area-turns of the $H$-coils are known within \pm 0.2 percent. The areas of the $B$-coils were determined in the same manner and to the same accuracy.

In calibrating the galvanometer for a test, the sensitivity can be adjusted to about 0.1 percent. Unless considerable care is taken, however, the error may be as great as 0.2 percent.

There is some difference of opinion with regard to the influence of galvanometer characteristics on the accuracy obtained. It is the authors' opinion, however, that a heavily overdamped galvanometer gives better results than a critically damped instrument, for this type of work.

It is important to avoid heating the specimen, especially if a high accuracy is desired. Since the present apparatus requires less than 600 watts to give 5,000 oersteds and has considerable radiating surface and heat capacity, there is practically no trouble from heating. Even after a rather long-continued test the temperature of the specimen does not rise more than about $2^{\circ} \mathrm{C}$.

The magnetic uniformity of the specimen is of particular importance if the results obtained with different permeameters which include different lengths of the specimen in the test are to be compared. With the present apparatus it is possible to investigate the uniformity of a specimen and thus determine whether or not it is suitable for use in intercomparisons.

One source of uncertainty, not heretofore considered to be characteristic of magnetically hard materials, is the phenomenon known as magnetic viscosity. This effect is noticeable in the range of magnetization represented by the steep parts of the normal induction and hysteresis curves. It was observed in Alnico and cobalt magnet steel and shown to be associated with the test specimens themselves and not to be brought about primarily by the rest of the magnetic circuit. It is proposed to study this effect further, as it may be the cause of some of the differences between the results obtained on the same specimens in different laboratories.

The observational error of a single reading may be as great as 0.2 or 0.3 percent. If a sufficient number of readings of the same quantity are taken, however, the average is probably good within 0.1 percent.

The uniformity of the field determines the limits of length and thickness of test coils which can be used in a given apparatus. The dimensions chosen for the coils in the present apparatus are such that errors due to nonuniform distribution do not exceed 0.2 percent.

The determination of values of magnetizing force corresponding to points on a hysteresis loop by means of a fixed $H$-coil involves taking the difference between two relatively large quantities. This may lead to large errors. By using a flip-coil, it is possible to determine directly the required value of magnetizing force and thus reduce materially the experimental error. The use of the flip-coil cuts down the amount of switching necessary and also makes it possible to check for initial polarization of the specimen by noting whether 
or not equal values of $H_{m}$ are obtained for the same value of magnetizing current flowing in either direction.

Taking into consideration all of the sources of error noted above, it is estimated that by the exercise of care in taking the readings and averaging several readings for each point, values of either induction or magnetizing force can be obtained which will be accurate within about 0.5 percent. For routine tests made without extreme precaution, values accurate within 1 percent should be obtained without difficulty.

\section{SUMMARY}

In order to meet the demand occasioned by the recent development of permanent-magnet alloys requiring the application of very high magnetizing forces for magnetizing and testing, a new permeameter has been constructed which has been called the NBS High- $H$ permeameter. In its design the aim has been to provide an instrument whose accuracy is sufficient to meet the requirements of presentday magnetic testing and which is simple and convenient to operate.

The fundamental principle is that of the well-known isthmus method, in which the magnetizing force is taken as the value of magnetic field at the surface of the specimen. By including only a relatively short length of specimen in the test, it is possible to apply the necessary high magnetizing forces without appreciable heating. Also, a symmetrical magnetic circuit promotes a uniform distribution of flux across the section of the specimen and a symmetrical distribution of field within the gap.

The requisite degree of accuracy is obtained by the use of test coils whose dimensions were chosen with reference to the observed distribution of flux and field in the magnetic circuit. Furthermore, by the use of a double $H$-coil it is possible to determine the value of the field at the surface of the specimen more accurately than can be done with a single coil. By using a rotating $H$-coil, it is possible in determining points on a hysteresis loop to avoid the errors which may arise from calculating the magnetizing force as the difference of two relatively large quantities.

It is estimated that for magnetizing forces from 100 to 5,000 oersteds it is possible under favorable conditions to obtain values of either induction or magnetizing force which will be accurate within 0.5 percent. Under ordinary conditions of routine testing the errors probably do not exceed 1 percent.

Washington, June 16, 1939. 